\title{
A Search for Late-time Radio Emission and Fast Radio Bursts from Superluminous Supernovae
}

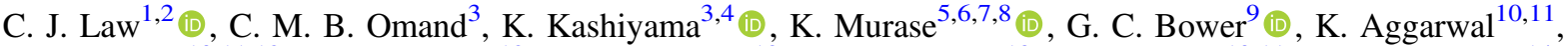 \\ S. Burke-Spolaor ${ }^{10,11,12}$ (D) B. J. Butler ${ }^{12}$ (i), P. Demorest ${ }^{12}$, T. J. W. Lazio ${ }^{13}$ (i) , J. Linford ${ }^{10,11}$, S. P. Tendulkar ${ }^{14}$, and \\ M. P. Rupen ${ }^{15}$ \\ ${ }^{1}$ Department of Astronomy and Radio Astronomy Lab, University of California, Berkeley, CA 94720, USA \\ ${ }_{2}^{2}$ Owens Valley Radio Observatory, California Institute of Technology, Pasadena, CA 91125, USA \\ ${ }^{3}$ Department of Physics, School of Science, the University of Tokyo, Tokyo 113-0033, Japan \\ ${ }^{4}$ Research Center for the Early Universe, the University of Tokyo, Tokyo 113-0033, Japan \\ ${ }^{5}$ Department of Physics, The Pennsylvania State University, University Park, PA 16802, USA \\ ${ }^{6}$ Department of Astronomy \& Astrophysics, The Pennsylvania State University, University Park, PA 16802, USA \\ ${ }^{7}$ Center for Particle and Gravitational Astrophysics, The Pennsylvania State University, University Park, PA 16802, USA \\ ${ }^{8}$ Yukawa Institute for Theoretical Physics, Kyoto University, Kyoto 606-8502, Japan \\ ${ }^{9}$ Academia Sinica Institute of Astronomy and Astrophysics, 645 N. A'ohoku Place, Hilo, HI 96720, USA \\ ${ }^{10}$ Department of Physics and Astronomy, West Virginia University, Morgantown, WV 26506, USA \\ ${ }^{11}$ Center for Gravitational Waves and Cosmology, West Virginia University, Chestnut Ridge Research Building, Morgantown, WV 26505, USA \\ ${ }^{12}$ National Radio Astronomy Observatory, Socorro, NM 87801, USA \\ 13 Jet Propulsion Laboratory, California Institute of Technology, Pasadena, CA 91109, USA \\ ${ }^{14}$ Department of Physics \& McGill Space Institute, McGill University, 3600 University Street, Montreal, QC H3A 2T8, Canada \\ ${ }^{15}$ National Research Council of Canada, Herzberg Astronomy and Astrophysics, Dominion Radio Astrophysical Observatory, P.O. Box 248, Penticton, BC V2A 6J9, \\ Canada \\ Received 2019 July 12; revised 2019 October 2; accepted 2019 October 2; published 2019 November 15
}

\begin{abstract}
We present results of a search for late-time radio emission and fast radio bursts (FRBs) from a sample of type-I superluminous supernovae (SLSNe-I). We used the Karl G. Jansky Very Large Array to observe 10 SLSN-I more than $5 \mathrm{yr}$ old at a frequency of $3 \mathrm{GHz}$. We searched fast-sampled visibilities for FRBs and used the same data to perform a deep imaging search for late-time radio emission expected in models of magnetar-powered supernovae. No FRBs were found. One SLSN-I, PTF10hgi, is detected in deep imaging, corresponding to a luminosity of $1.2 \times 10^{28} \mathrm{erg} \mathrm{s}^{-1}$. This luminosity, considered with the recent $6 \mathrm{GHz}$ detection of PTF10hgi in Eftekhari et al., supports the interpretation that it is powered by a young, fast-spinning ( $\sim \mathrm{ms}$ spin period) magnetar with $\sim 15 M_{\odot}$ of partially ionized ejecta. Broadly, our observations are most consistent with SLSNe-I being powered by neutron stars with fast spin periods, although most require more free-free absorption than is inferred for PTF10hgi. We predict that radio observations at higher frequencies or in the near future will detect these systems and begin constraining properties of the young pulsars and their birth environments.
\end{abstract}

Unified Astronomy Thesaurus concepts: Extragalactic radio sources (508); Radio bursts (1339); Supernovae (1668); Radio interferometry (1346)

\section{Introduction}

The advent of wide-field surveys focused on the time domain has led to the discovery and characterization of new, rare classes of transient astrophysical phenomena. Optical surveys have identified extremely luminous classes of transients called superluminous supernovae (SLSNe; Gal-Yam 2012). The hydrogenpoor subset of SLSNe ("type-I") are unlikely to be powered by interaction with their circumburst medium. This suggests that something powers them internally, such as an accreting black hole (Woosley \& Bloom 2006) or rapidly spinning young neutron star (Murase et al. 2016; Metzger et al. 2017).

At centimeter-wavelengths, radio surveys have identified the fast radio burst (FRB; Cordes \& Chatterjee 2019; Petroff et al. 2019), a coherent, millisecond transient. The recent association of an FRB with a galaxy at $z=0.1927$ confirmed that they are extremely bright and luminous (Chatterjee et al. 2017; Tendulkar et al. 2017), motivating new models for FRB origin (Kashiyama \& Murase 2017). A new suite of FRB origin models has already been published (Metzger et al. 2019). However, only one model has successfully predicted the properties of FRB 121102: young magnetars (Murase et al. 2016).
Newborn magnetars have emerged as a strong candidate for producing a variety of luminous transients (e.g., Maeda et al. 2007; Metzger et al. 2015; Nicholl et al. 2015; Kashiyama et al. 2016; Margalit et al. 2018). Classes of objects such as SLSNeI, FRBs, and even ultralong gamma-ray bursts (GRBs) have severe energetic requirements that can be met by tapping into the spin-down power of a magnetar with a millisecond rotation period. The magnetar birth scenario presents a testable hypothesis: SLSNe-I should be associated with luminous pulsar wind nebulae (PWNe) at late times (Murase et al. 2016; Metzger et al. 2017; Omand et al. 2018). It is also possible that SLSNe-I leave compact remnants that emit coherent radio emission detectable as FRBs. Coherent radio emission from pulsars is observationally well characterized and the fraction of sources detectable by this emission is roughly 10\% (Tauris \& Manchester 1998).

Eftekhari et al. (2019) found the first observational support for the magnetar-powered supernova model with the detection of late-time radio emission coincident with the SLSN-I known as PTF10hgi. The radio source is located in a dwarf galaxy, similar to that seen for most SLSNe-I (Lunnan et al. 2014; Vreeswijk et al. 2014), but it could also potentially be 
Table 1

SLSN-I Sample

\begin{tabular}{|c|c|c|c|c|}
\hline Name & Redshift & $\begin{array}{l}\text { R.A. } \\
\text { (J2000) }\end{array}$ & $\begin{array}{c}\text { Decl. } \\
(\mathrm{J} 2000)\end{array}$ & $\begin{array}{l}\text { Age } \\
(\mathrm{yr})\end{array}$ \\
\hline SN 2005ap ${ }^{a}$ & 0.283 & $13: 01: 14: 83$ & $+27: 43: 32: 3$ & 9.9 \\
\hline SN 2007bi & 0.127 & $13: 19: 20: 14$ & $+08: 55: 43: 7$ & 9.4 \\
\hline SN 2006oz & 0.396 & $22: 08: 53: 56$ & $+00: 53: 50: 4$ & 8.0 \\
\hline PTF10hgi ${ }^{b}$ & 0.098 & $16: 37: 47: 04$ & $+06: 12: 32: 3$ & 6.8 \\
\hline PTF09cnd & 0.258 & $16: 12: 08: 94$ & $+51: 29: 16: 1$ & 6.6 \\
\hline SN 2010kd & 0.101 & 12:08:00:89 & $+49: 13: 32: 9$ & 6.4 \\
\hline SN $2010 g^{c}$ & 0.23 & $11: 25: 46: 71$ & $-08: 49: 41: 4$ & 6.2 \\
\hline PTF09cwl & 0.349 & $14: 49: 10: 08$ & $+29: 25: 11: 4$ & 6.1 \\
\hline SN 2011ke & 0.143 & $13: 50: 57: 77$ & $+26: 16: 42: 8$ & 5.7 \\
\hline PTF09atu & 0.501 & $16: 30: 24: 55$ & $+23: 38: 25: 0$ & 5.5 \\
\hline
\end{tabular}

Notes.

${ }^{\text {a }}$ Late-time radio limit at $1.4 \mathrm{GHz}$ by Schulze et al. (2018).

${ }^{\mathrm{b}}$ Late-time radio detection at $6 \mathrm{GHz}$ by Eftekhari et al. (2019).

${ }^{\mathrm{c}}$ Late-time radio limit at $3 \mathrm{GHz}$ by Hatsukade et al. (2018).

associated with an AGN. It is also possible that the emission is associated with the afterglow of an off-axis jet of a GRB. New observations to constrain the temporal and spectral evolution of the source will help distinguish between these classes of objects. Late radio observations have been a powerful tool for studying long GRBs (Soderberg et al. 2004), short GRBs (Metzger \& Bower 2014), and tidal disruption events (Bower et al. 2013).

Here we present a multifaceted search for signatures of magnetar birth in SLSNe-I. We use the Karl G. Jansky Very Large Array (VLA) to search for late-time radio emission at $3 \mathrm{GHz}$ that is coincident with known SLSNe-I and use the results for detailed modeling of magnetar birth models. We detect 1 of the 10 sources, PTF10hgi, confirming work presented in Eftekhari et al. (2019). We used the real-time transient search system known as realfast to commensally search for FRBs in the same data through millisecond imaging (Law et al. 2018).

\section{Data and Analysis}

\subsection{Observations}

We used the VLA to observe a sample of 10 SLSN-I at $3 \mathrm{GHz}$. We selected the oldest 10 SLSN-I from the first large sample with well-characterized host galaxies (Lunnan et al. 2014). This sample has rest-frame ages greater than $5 \mathrm{yr}$, but excludes SCP 06F6, as it is predicted to be too faint to detect in a reasonable amount of time. Table 1 lists the SLSN-I in order of their rest-frame age at time of observation in late 2017. The VLA observations were designed with two goals: search for late-time radio emission and search for FRBs. The late-time radio emission from magnetar-powered supernovae is expected to fade as $t^{-2}$, but is also subject to free-free absorption by the supernova ejecta at early times (e.g., Kashiyama \& Murase 2017). The balance of these two effects favors observations at frequencies from 2 to $10 \mathrm{GHz}$ on timescales of 5-20 yr. For the FRB search, we favored observing frequencies $\lesssim 3 \mathrm{GHz}$, where most FRBs have been observed. At lower frequencies, the VLA has a larger field of view, which also improves the odds of detecting an FRB that is unassociated with the SLSN-I.

We observed with the $3 \mathrm{GHz}$ band as a compromise between the expected late-time emission and FRB detection goals. We
Table 2

Observations of SLSN-I

\begin{tabular}{lccc}
\hline \hline Name & $\begin{array}{c}\text { Epochs } \\
(\mathrm{MJD})\end{array}$ & $\begin{array}{c}\text { Obs. Time } \\
(\mathrm{min} ; \text { total })\end{array}$ & $\begin{array}{c}\text { Sensitivity } \\
\left(\mu \mathrm{Jy} \mathrm{beam}^{-1} ; 1 \sigma\right)\end{array}$ \\
\hline SN 2005ap & 58060,58131 & 57 & 10 \\
SN 2007bi & 58074,58128 & 34 & 22 \\
SN 2006oz & 58036,58124 & 60 & 8 \\
PTF10hgi $^{\mathrm{a}}$ & 58045,58130 & 26 & 14 \\
PTF09cnd & 58045,58130 & 46 & 11 \\
SN 2010kd & 58074,58128 & 27 & 14 \\
SN 2010gx & 58074,58128 & 41 & 11 \\
PTF09cwl & 58060,58131 & 73 & 8 \\
SN 2011ke & 58060,58131 & 35 & 12 \\
PTF09atu & 58045,58130 & 109 & 8 \\
\hline
\end{tabular}

Note.

${ }^{\mathrm{a}}$ Detection with peak flux density of $47 \mu \mathrm{Jy}$.

used eight spectral windows covering the full frequency range from 2.5 to $3.5 \mathrm{GHz}$ using 32 channels per window with a width of $4 \mathrm{MHz}$ per channel. The visibility data were recorded with $5 \mathrm{~ms}$ cadence (comparable to FRB pulse width; Petroff et al. 2016) to allow a real-time search for FRBs with realfast. ${ }^{16}$ The antennas were in the " $\mathrm{B}$ " configuration, which has baseline lengths up to $10 \mathrm{~km}$ and a synthesized beam size of roughly $3^{\prime \prime}$ at $3 \mathrm{GHz}$. These data are thus sensitive to FRBs anywhere within the primary beam, which has a full width at half power of $14^{\prime}$.

Table 2 describes the observations of each target. The 10 targets were scheduled in four groups, each of which was observed in two epochs from late 2017 to early 2018. The observing duration for each epoch was set to detect a source with a power roughly 10 times lower than the persistent radio source associated with FRB 121102 ( $3 \sigma$ power sensitivity of $L_{3 \mathrm{GHz}}=3 \times 10^{28} \mathrm{erg} \mathrm{s}^{-1} \mathrm{~Hz}^{-1}$ ).

For three of the observing epochs (MJD 58128, 58130, and 58131), the correlator was not able to write data fast enough, so some data were lost. Roughly $\sim 20 \%$ of data were affected by correlator issues, interference, or bad calibration solutions; in some later observations, up to $50 \%$ of data were lost.

\subsection{Fast Transient Search}

After each observation, we searched the $5 \mathrm{~ms}$ data for FRBs with the rfpipe search pipeline (Law 2017). The search was run offline using CPUs in spare nodes of the VLA correlator cluster. This search applies calibration solutions calculated in real time by the VLA observing system (a.k.a. telcal). Bad channels and integrations are flagged using a sigma clipping algorithm, while the variance of visibilities over baselines is used to flag near-field interference for specific channelintegration-polarization bins.

We searched for FRBs with dispersion measures up to $3000 \mathrm{pc} \mathrm{cm}^{-3}$ and pulse widths up to $40 \mathrm{~ms}$. The maximum distance for this SLSN-I sample is $z \sim 0.5$ which implies DM contribution from the intergalactic medium of roughly $400 \mathrm{pc} \mathrm{cm}^{-3}$ (Prochaska \& Zheng 2019). The DM contribution from the Milky Way is smaller than the extragalactic contribution in all cases (Cordes \& Lazio 2002). The DM contribution from the FRB environment and host galaxy is generally expected to be less than $3000 \mathrm{pc} \mathrm{cm}^{-3}$ (Kulkarni et al. 2014); in the case of FRB

${ }^{16}$ See also http://realfast.io. 
121102, this component contributes less than $225 \mathrm{pc} \mathrm{cm}^{-3}$ to the total DM measurement (Tendulkar et al. 2017).

All candidates brighter than $8 \sigma$ were inspected by looking at dedispersed burst spectra and $5 \mathrm{~ms}$ image associated with the event. No bursts were found brighter than $8 \sigma$. A typical observation had 26 antennas and $1.5 \mathrm{GHz}$ of clean bandwidth, which corresponds to a sensitivity of roughly $4 \mathrm{mJy}$ per $5 \mathrm{~ms}$ snapshot image.

The nominal sensitivity is idealized and needs to be corrected for the effects of dedispersion and primary beam attenuation. The rfpipe search uses a brute-force dedispersion algorithm that can lose sensitivity to pulses with DM between the DM search grid (Keane \& Petroff 2015). The DM search grid was set to lose at most $5 \%$ of the nominal sensitivity due to intra-DM sensitivity losses, so the $8 \sigma$ limit is thus $34 \mathrm{mJy}$ in 5 $\mathrm{ms}$ at the center of the primary beam. The image search was also sensitive to FRBs throughout the primary beam, which has an FWHM of $14^{\prime}$ at $3 \mathrm{GHz}$. The search for FRBs throughout the primary beam was complete to a flux limit of $68 \mathrm{mJy}$ in 5 ms. The sensitivity is best defined as a fluence limit averaged over the observing band from 2.5 to $3.5 \mathrm{GHz}$, so sensitivity to temporally or spectrally narrow emission structure is worse than stated here (Law et al. 2017; Gajjar et al. 2018).

\subsection{Deep Imaging}

We averaged the $5 \mathrm{~ms}$ integrations to $1 \mathrm{~s}$ and analyzed these new data sets with the CASA calibration pipeline (McMullin et al. 2007). Any $1 \mathrm{~s}$ integration with more than $30 \%$ of its subintegrations flagged was fully flagged.

Three of the observing blocks (including seven of the targets) used a standard flux calibrator (either 3C286 or 3C48). These fields were calibrated with the VLA CASA calibration pipeline (version 5.4.0). One observing block, including PTF09atu, PTF09cnd, and PTF10hgi, used 3C295 as a flux calibrator, which is not supported by the latest pipeline. For these observations, we instead used the VLA scripted pipeline (version 1.4.0). In all cases, calibration quality was validated by inspecting the standard pipeline output of calibrator images, gain solutions, and visibility plots.

Both epochs of all 10 fields were imaged with tclean in CASA. For each field, we first produced a sky model through a light clean of mJy-brightness sources using natural weighting. In some cases, that model was sufficient to self-calibrate the field at both epochs with a single solution per antenna and spectral window (Stokes $I$ ). We then created a final map for each field by combining both epochs and creating a deeply cleaned image. For images with image artifacts from nearby sources, we use robust weighting of 0.5 . The best images from either natural or robust weighting were used to estimate noise and search for radio emission from the SLSN.

Table 2 lists the measured sensitivity of a deep image made for each SLSN. Only one of the targets, PTF10hgi is detected with greater than $3 \sigma$ significance. For three of the fields (PTF09cnd, SN2007bi, and SN2006oz), we detected a radio source within $1^{\prime}$ of the SLSN. However, all of these radio sources are offset by more than $10^{\prime \prime}$ (far larger than any astrometric uncertainty), which makes them highly unlikely to be associated with the SLSN or their host galaxies (host galaxy images at Lunnan et al. 2014). Stacking all 10 images by the inverse noise squared gives an image with no significant source at the location of the SLSN-I and a $3 \sigma$ limit of $8 \times 10^{-7} \mathrm{Jy}$.

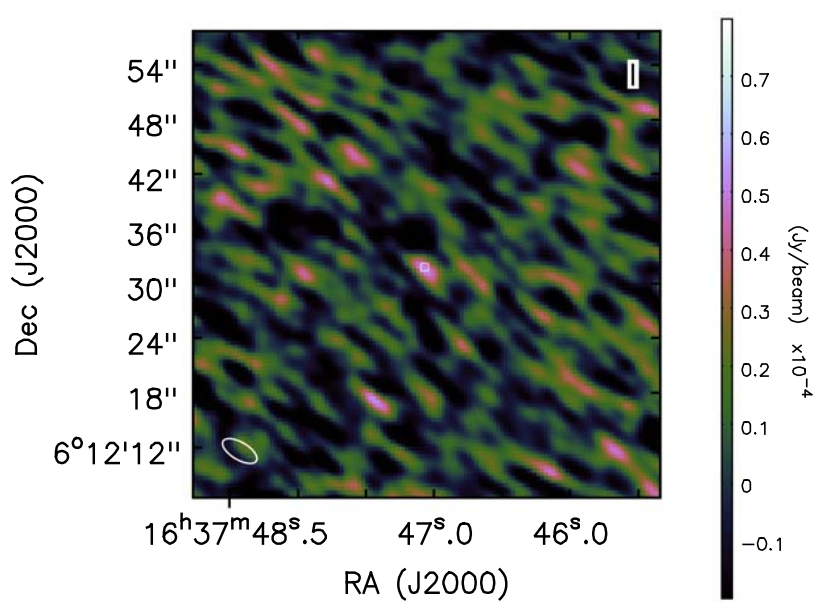

Figure 1. VLA $3 \mathrm{GHz}$ radio image of PTF10hgi. The location of the $6 \mathrm{GHz}$ source associated with PTF10hgi is shown with a white square (Eftekhari et al. 2019).

Figure 1 shows a compact source at the location of the $6 \mathrm{GHz}$ counterpart to PTF10hgi (Eftekhari et al. 2019). This source is apparent in both observing epochs and is robust to a range of assumptions for imaging and self-calibration parameters. We modeled this region with the CASA imfit tool to define a $2 \mathrm{~d}$ Gaussian with width fixed to the synthesized beam shape. The best-fit source has a peak flux density of $47 \pm 14 \mu \mathrm{Jy}$ located at (R.A., decl.) $(\mathrm{J} 2000)=(16: 37: 47.04$, $6: 12: 31.4)$ with centroid uncertainty of 0 ." $7 \times 0$ " 4 . The $3 \mathrm{GHz}$ radio source is coincident with the $6 \mathrm{GHz}$ source located at $(16: 37: 47.071,6: 12: 31.88)$. A $3 \sigma$ detection significance corresponds to a false alarm rate of $10^{-3}$ or a $1 \%$ probability of detection in a sample of 10 sources. Using the observed noise properties of the PTF10hgi $3 \mathrm{GHz}$ image, we estimate a smaller than 1 in 100 chance of false association with this source.

The $6 \mathrm{GHz}$ source is consistent with the optical position of PTF10hgi (Lunnan et al. 2014), but the $3 \mathrm{GHz}$ location is offset roughly $1^{\prime \prime}$ from optical position. We attribute this to small phase calibration errors, which can affect localizations smaller than the $3^{\prime \prime}$ synthesized beam size. Uncertainties in referencing the optical and radio frames may also contribute. Hereafter, we assume that the 3 and $6 \mathrm{GHz}$ sources are coincident with each other and the SLSN-I.

The $3 \mathrm{GHz}$ flux density of PTF10hgi corresponds to a luminosity $L_{\nu}=(1.2 \pm 0.4) \times 10^{28} \mathrm{erg} \mathrm{s}^{-1} \mathrm{~Hz}^{-1}$. Observations of PTF10hgi at 3 and $6 \mathrm{GHz}$ were made within three months of each other, so they are effectively simultaneous in the context of synchrotron emission models (see Section 3). Comparing this $3 \mathrm{GHz}$ flux density to the $6 \mathrm{GHz}$ measurement implies a spectral index $\alpha=0.0 \pm 0.6\left(F_{\nu} \propto \nu^{\alpha}\right)$. This spectral index measurement is consistent with, and slightly more precise than, that of Eftekhari et al. (2019).

\section{Discussion}

This study is the first search for late-time radio emission and FRBs from a sample of SLSN-I. There are only three SLSN-I with prior observational constraints on late-time $(>5 \mathrm{yr})$ radio emission. Schulze et al. (2018) present an upper limit of $F_{1.4 \mathrm{GHz}}<75 \mu \mathrm{Jy}(3 \sigma)$ for SN2005ap roughly $10 \mathrm{yr}$ after explosion, Hatsukade et al. (2018) present $3 \mathrm{GHz}$ upper limits on $8 \mathrm{SLSNe}$ (5 of which were SLSNe-I, one of which is in our 

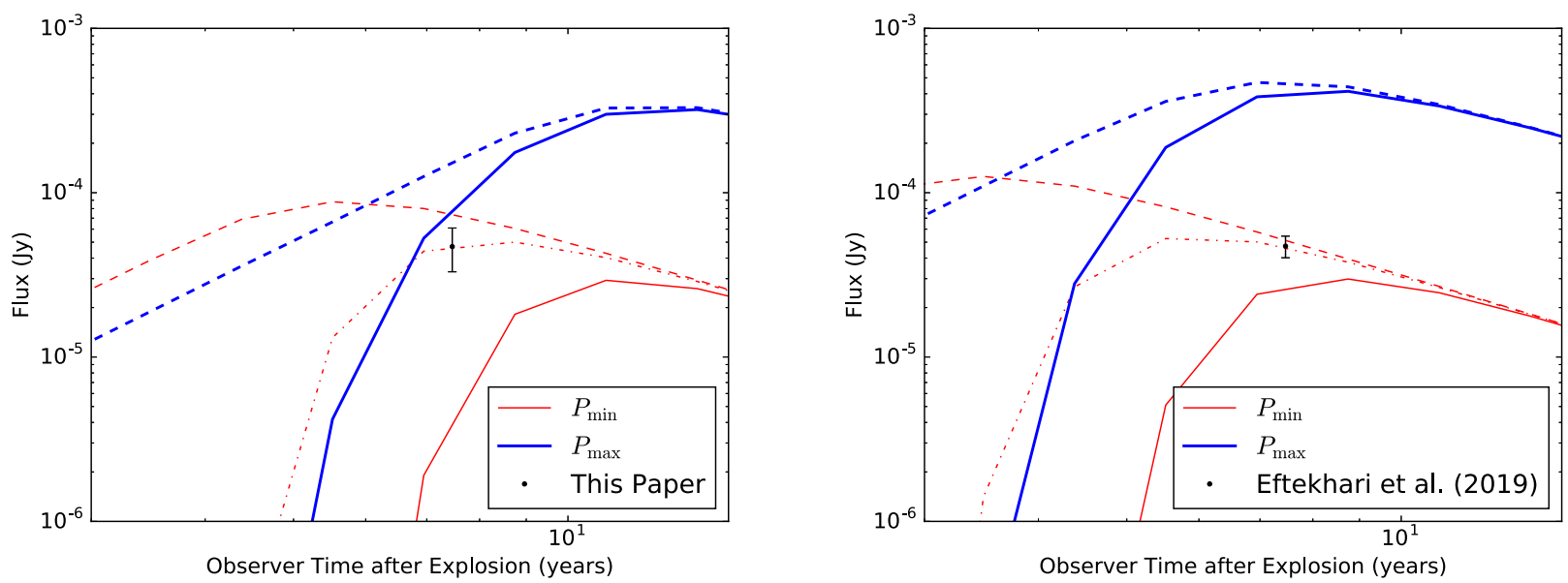

Figure 2. Detected fluxes for PTF10hgi at $3 \mathrm{GHz}$ (left panel; this paper) and $6 \mathrm{GHz}$ (right panel; Eftekhari et al. 2019) with their $1 \sigma$ uncertainties shown in black. The $P_{\min }(1 \mathrm{~ms})$ and $P_{\max }$ models shown in Table 3 are displayed in red and blue, respectively, with the solid lines indicating the light curve with absorption and dashed lines indicating the curve with no absorption. We find that a $P_{\min }$ model with $30 \%-50 \%$ of the ejecta singly ionized, with the rest neutral, can reproduce the observed data; the dashed-dotted line for $P_{\min }$ indicates a model with $40 \%$ of the ejecta ionized.

sample), and Eftekhari et al. (2019) detect PTF10hgi with a flux of $F_{6 \mathrm{GHz}}=47.3 \pm 7.1 \mu \mathrm{Jy}$ roughly $7 \mathrm{yr}$ after explosion.

\subsection{Modeling}

In a magnetar-powered supernova, the persistent radio source luminosity is defined by the magnetar-driven wind interacting with its surrounding supernova remnant (Murase et al. 2016; Metzger et al. 2017; Omand et al. 2018). The magnetar birth properties (especially initial spin period and magnetic field strength) are inferred from the early optical light curve. We use optical data from the Open Supernova catalog ${ }^{17}$ (Guillochon et al. 2017) and fit by eye with a three-parameter model (Kashiyama et al. 2016): the initial spin period $P$ and the magnetic field $B_{13}=B /\left(10^{13} \mathrm{G}\right)$ of the neutron star, and the mass $M_{\mathrm{ej}}$ of the supernova ejecta. Using this method, we can determine the parameters to within 5\%-10\%. Since the magnetar model has degeneracies, we define one parameter set with $P=1 \mathrm{~ms}\left(P_{\min }\right)$, which is close to the mass-shedding limit for neutron stars (Watts et al. 2016) and another with a larger period $\left(P_{\max }\right)$, which is the largest spin period consistent with the optical light curve. Omand et al. (2018) finds that $P_{\max }$ varies between supernovae, but is typically less than $5 \mathrm{~ms}$.

There are multiple approaches to modeling optical light curves of SLSNe-I (e.g., Inserra et al. 2013; Liu et al. 2017; Nicholl et al. 2017; Prajs et al. 2017) and they sometimes derive different magnetar parameters for the same sources. Earlier studies tended to assume a simple dipole spin-down model, while we use a model based on numerical simulation (Gruzinov 2005; Spitkovsky 2006; Tchekhovskoy et al. 2013). The numerical simulations require much smaller $B_{13}$ and $P$ for a given spin-down luminosity (Kashiyama et al. 2016). Our treatment also allows for acceleration of the ejecta due to interaction with the PWN, which couples the dynamics of the ejecta to the spin-down luminosity; a realistic ejecta profile (a homologous core; Kasen \& Bildsten 2010); and self-consistently treats the radio and optical signatures to break degeneracies inherent to the optical data alone.

Once the magnetar parameters have been found, we calculate the time evolution of the radio emission from the PWN based on these optically derived parameters. This emission from the

\footnotetext{
${ }^{17}$ https://sne.space/
}

PWN is calculated as in previous papers (see Gaensler \& Slane 2006; Tanaka \& Takahara 2010, and references therein). We model not only the dynamics of PWNe and SNe as in our three-parameter optical model, but also self-consistently calculate pair cascades, Compton and inverse Compton scattering, adiabatic cooling and both internal and external attenuation by solving the Boltzmann equation for electron/ positrons and photons in the PWN over all electron energies and photon frequencies (Murase et al. 2015, 2016). We assume an electron-positron injection spectrum motivated by Galactic PWNe such as the Crab PWNe (e.g., Tanaka \& Takahara 2010, 2013), a broken power law with a peak Lorentz factor of $\gamma_{b}=10^{5}$ and injection spectral indices of $q_{1}=1.5$ and $q_{2}=2.5$. Free-free absorption in the ejecta is calculated assuming a singly ionized oxygen ejecta, and we do not consider absorption outside the ejecta.

\subsection{PTF10hgi}

Given the new, more precise, spectral index measurement, we discuss the viability of different astrophysical models for PTF10hgi. As discussed in Eftekhari et al. (2019), there are three viable models for this radio emission: an AGN, an offaxis GRB jet, and a nebula produced by a remnant magnetar. The spectral index is consistent with radio-loud AGN (Elvis et al. 1994; Chatterjee et al. 2017), but the AGN scenario is unlikely (Eftekhari et al. 2019) because either the black hole would have an unexpectedly large mass for a radio-quiet AGN (5\% of the host galaxy, while dwarf galaxy black holes are generally $\leq 0.1 \%$ of the total mass Merloni et al. 2003; Reines et al. 2013) or the host galaxy would be peculiar, because the prevalence of radio-loud AGN in dwarf galaxies is $\lesssim 1 \%$ (Reines et al. 2013). The off-axis GRB model predicts bright emission at earlier times, well above the limits placed on other SLSNe (Coppejans et al. 2018; Eftekhari et al. 2019), and predicts a spectral index $\alpha \sim-1$, which is disfavored by our observations.

The magnetar model predictions for the $P_{\min }$ and $P_{\max }$ cases, along with the data at 3 and $6 \mathrm{GHz}$, are shown in Figure 2. The $P_{\max }$ model slightly overpredicts the data at $3 \mathrm{GHz}$, but severely overpredicts at $6 \mathrm{GHz}$, while the $P_{\min }$ model slightly underpredicts at both frequencies with absorption, but is close to fitting both points with little or no absorption. We find that a 

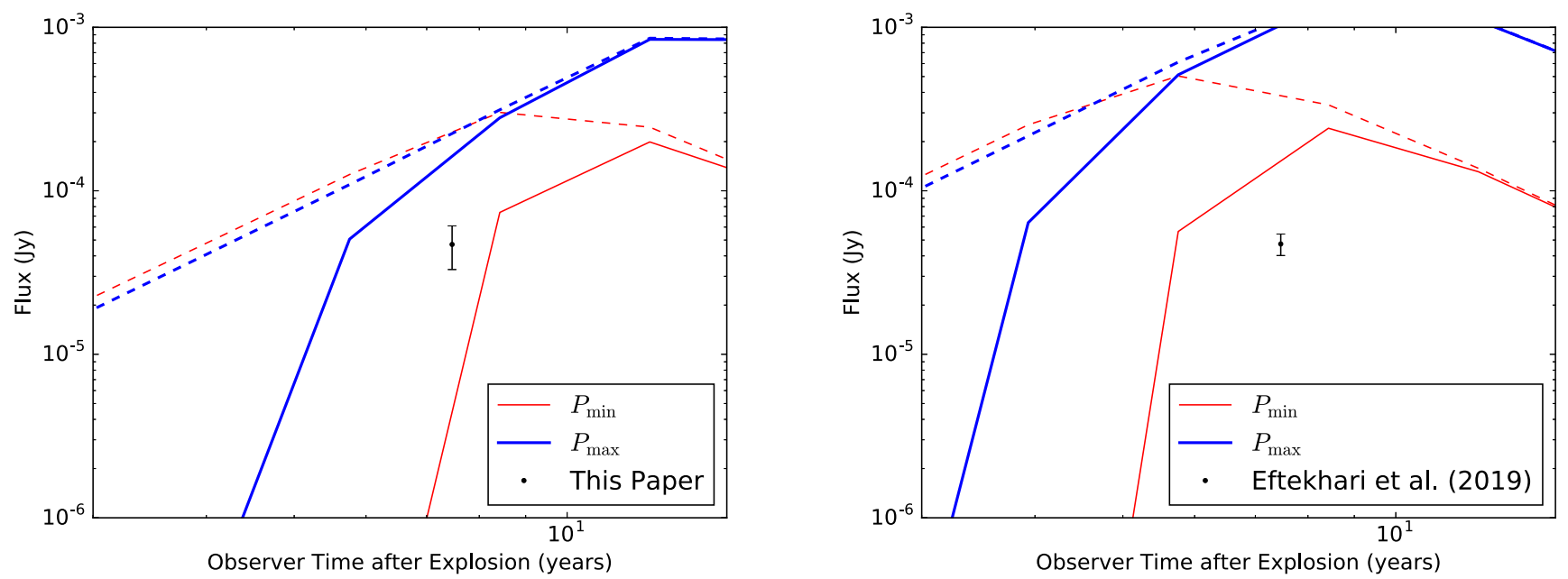

Figure 3. Same as Figure 2, but with $\gamma_{b}=10^{2}$ instead of $10^{5}$.

Table 3

Model Parameters from Fits to the Optical Light Curves for Those SLSNe-I with Sufficient Optical Data to Constrain Those Models

\begin{tabular}{|c|c|c|c|c|c|c|}
\hline Name & $\begin{array}{c}B_{13} \text { at } 1 \mathrm{~ms} \\
\text { (G) }\end{array}$ & $\begin{array}{c}M_{\mathrm{ej}} \text { at } 1 \mathrm{~ms} \\
\left(M_{\odot}\right)\end{array}$ & $\begin{array}{l}P_{\max } \\
(\mathrm{ms})\end{array}$ & $\begin{array}{c}B_{13} \text { at } P_{\max } \\
\text { (G) }\end{array}$ & $\begin{array}{c}M_{\mathrm{ej}} \text { at } P_{\max } \\
\left(M_{\odot}\right)\end{array}$ & Data Reference \\
\hline SN2005ap & 3.0 & 7 & 1.4 & 2.0 & 2.0 & Quimby et al. (2007) \\
\hline SN2007bi & 4.0 & 25 & 2.2 & 2.0 & 5.5 & Gal-Yam et al. (2009) \\
\hline SN2006oz & 5.0 & 12.5 & 2.0 & 2.0 & 2.5 & Leloudas et al. (2012) \\
\hline PTF09cnd & 2.0 & 14 & 1.0 & 2.0 & 14 & Quimby et al. (2011) \\
\hline PTF10hgi & 14 & 15 & 4.2 & 4.0 & 2.0 & Inserra et al. (2013) \\
\hline SN2010kd & 4.7 & 25 & 2.4 & 2.0 & 4.0 & Vinko et al. (2012) \\
\hline SN2010gx & 4.5 & 10.0 & 1.6 & 3.5 & 3.5 & Pastorello et al. (2010) \\
\hline PTF09cwl & 2.0 & 12 & 1.5 & 1.7 & 3.5 & Brown et al. (2014) \\
\hline SN2011ke & 7.5 & 9.5 & 2.4 & 2.9 & 1.3 & Inserra et al. (2013) \\
\hline PTF09atu & 3.0 & 14 & 1.6 & 2.0 & 4.5 & Yaron \& Gal-Yam (2012) \\
\hline
\end{tabular}

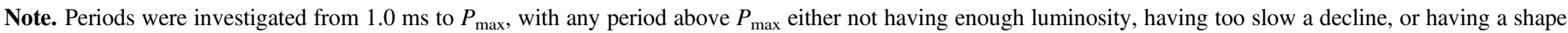
inconsistent with the observed data. The uncertainty on these parameters is $5 \%-10 \%$ each.

$P_{\text {min }}$ model with $30 \%-50 \%$ of the ejecta singly ionized, with the rest neutral, can reproduce the observed data; a model with $40 \%$ ionization is shown in Figure 2. This might be expected for a large ejecta mass, or the lack of absorption could mean that the ejecta are clumped in some regions away from the line of sight and relatively unobstructed along the line of sight. The $P_{\text {min }}$ model also disfavors a Wolf-Rayet progenitor, as the ejecta mass is larger than expected in that model.

We can also use this detection to constrain the electroninjection spectrum. The injection Lorentz factor $\gamma_{b}$, which governs the frequency of the spectral break where most of the energy is injected, cannot be constrained in optical observations, as they are only sensitive to the total energy injected, and could take values from $10^{2}$ to $10^{6}$. Figure 2 shows models with $\gamma_{b}=10^{5}$, which is also assumed in Omand et al. (2018); this means the $\nu F_{\nu}$ synchrotron spectrum peaks at UV/X-ray energies. Figure 3 shows the light curves for the same parameters, except with $\gamma_{b}=10^{2}$, giving a spectrum that peaks at infrared/microwave energies-models for the persistent emission from FRB 121102 usually have spectra that peak in this range (Margalit \& Metzger 2018; C. M. B. Omand et al. 2019 , in preparation). We see that the luminosity at peak in the radio bands is much higher, completely excluding all models at $6 \mathrm{GHz}$, even though the light curve has yet to reach its peak. Based on this result, we show that these results favor higher values of $\gamma_{b}$, and exclude those with $\gamma_{b} \lesssim 10^{4}$.
Overall, the detections in both bands are most consistent with the magnetar model, but observations at more frequencies and epochs will be needed to determine the system properties with any certainty. Models involving fast cooling emission predict a strong evolution in the spectral index through its peak luminosity, up until there is a consistent, negative spectral index ( $\alpha=-1 / 2$; C. M. B. Omand et al. 2019, in preparation). Models involving relic cooling emission predict a weak evolution of the spectral index, remaining almost flat even after peak (e.g., Margalit \& Metzger 2018; Omand et al. 2018). The measured flat spectral index suggests that PTF10hgi may be near peak and disfavors a detection early in the rise of the radio emission, but only further observations can differentiate the two scenarios.

\subsection{Nondetections}

Figure 4 shows the $3 \sigma$ upper limit on luminosity for the higher sensitivity observation for undetected SLSN shown in Table 2 as a function of time since explosion. Table 3 lists the magnetar and ejecta parameters that fit the optical light curves and are used for modeling radio emission.

The observational constraints on these models, which are summarized in Table 4, are as follows:

1. PTF09atu: We were not able to exclude any of the models, even those with no absorption. This is likely 

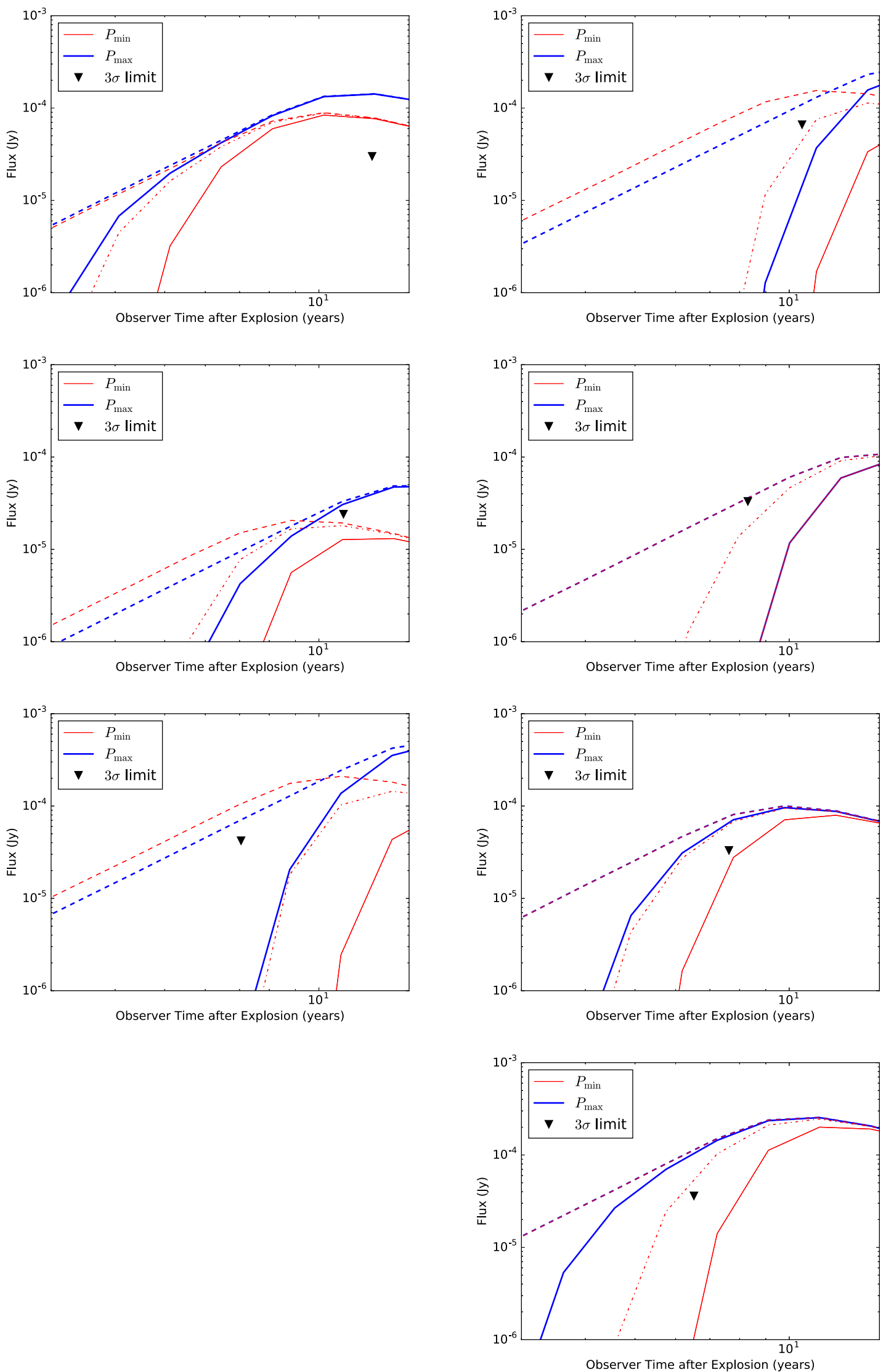

Figure 4. Comparison of observed $3 \mathrm{GHz}$ flux limits and expected flux from a range of pulsar-driven models constrained by the optical emission. Each panel shows a specific SLSNe-I, ordered from left to right, starting at top, as SN2005ap, SN2007bi, SN2006oz, PTF09cnd, SN2010kd, SN2010gx, PTF09cwl, SN2011ke, and PTF09atu. The black triangle shows the $3 \sigma$ flux limit, the red and blue lines show the models assuming $P_{\min }$ and $P_{\max }$ parameter sets, respectively, and the solid lines indicate the modeled radio flux assuming absorption, the dashed lines indicate the curve with no absorption, and the dashed-dotted $P_{\min }$ line indicating a model with $40 \%$ ionization. 
Table 4

A Summary of Viability of Parameter Sets for Radio Flux Calculations

\begin{tabular}{lccccc}
\hline \hline Name & $P_{\min }$ abs & $P_{\min }$ unabs & $P_{\max }$ abs & $P_{\max }$ unabs & $P_{\min } \mathrm{w} / \mathrm{PTF} 10 \mathrm{hgi-like}$ abs \\
\hline SN2005ap & Excluded & Excluded & Excluded & Excluded & Excluded \\
SN2007bi & Viable & Excluded & Viable & Excluded & Viable \\
SN2006oz & Viable & Viable & Excluded & Excluded & Viable \\
PTF09cnd & Viable & Excluded & Viable & Excluded & Viable \\
SN2010kd & Viable & Excluded & Viable & Excluded & Excluded \\
SN2010gx & Viable & Excluded & Excluded & Excluded & Viable \\
PTF09cwl & Viable & Excluded & Viable & Excluded & Excluded \\
SN2011ke & Viable & Excluded & Excluded & Excluded & Viable \\
PTF09atu & Viable & Viable & Viable & Viable & \\
\hline
\end{tabular}

Note. Sets of models were made for $P_{\min }$ and $P_{\max }$ and minimal and maximal free-free absorption opacity, as well as for PTF10hgi-like absorption.

because PTF09atu is the furthest and youngest SLSNe-I in our sample.

2. SN2007bi, PTF09cnd, SN2010kd, and PTF09cwl: Models with absorption are still viable to explain these SLSNe, but models with no absorption are excluded. The amount of absorption needed to be consistent with the model varies by supernova; PTF09cnd and PTF09cwl both need only a small amount of absorption to be consistent, while SN2007bi and SN2010kd both require more. All of them are consistent with a $P_{\min }$ model with $40 \%$ ionized ejecta, like PTF10hgi.

3. SN2010gx and SN2011ke: Both of these supernovae exclude models without absorption as well, and the $P_{\min }$ model would require a large amount of absorption in order to be consistent with observations, more than the best-fit model for PTF10hgi. The $P_{\max }$ model is also completely excluded for these two supernovae, so a faster spinning pulsar with larger magnetic field and ejecta mass is required to be consistent.

4. SN2006oz: Free-free absorption here is predicted to be small, regardless of the pulsar parameters, mostly due to the age of the system. The $P_{\max }$ model is excluded by these observations, while the $P_{\text {min }}$ model is still viable, even though the emission is predicted to be at or after the peak. Only a small reduction in period from the $P_{\max }$ model would be required to make the model viable, however, since the predicted emission has almost the same flux as our $3 \sigma$ limit.

5. SN2005ap: None of the models are consistent with our observations, as they all overpredict the expected emission. There are three likely reasons for this: this SLSN-I is not magnetar-driven; the electron-injection spectrum is not broad and Crab-like (e.g., Tanaka \& Takahara 2010, 2013), but sharply peaked at higher energies (C. M. B. Omand et al. 2019, in preparation); or the ejecta are more heavily ionized than predicted. Margalit et al. (2018) predicts at most singly ionized species, but assumes $10 M_{\odot}$ of ejecta. However, SN2005ap is best modeled with 2-7 $M_{\odot}$ of ejecta and Milisavljevic et al. (2018) finds evidence for higher oxygen lines in SN2012au, a putative magnetar-driven supernova. Given these points, the ejecta may become more ionized on a timescale of $\sim 5 \mathrm{yr}$. Free-free absorption outside the ejecta could also suppress the emission further.

\section{Conclusions}

We reported on new VLA observations to test the hypothesis that SLSNe-I are powered by young pulsars or magnetars. Of the 10 SLSNe-I observed, we detect one, PTF10hgi, which supports earlier results and the argument it is a magnetarpowered supernova (Eftekhari et al. 2019). The detections of PTF10hgi are most consistent with the fastest-spinning magnetar model with minimal free-free absorption, for microphysical parameters similar to those of Galactic pulsar wind nebula (Murase et al. 2016; Omand et al. 2018). The detection is also inconsistent with models with a low electroninjection Lorentz factor, which is typical for models of the persistent source of FRB 121102. This may imply that these two sources have different electron acceleration mechanisms, or that the acceleration mechanism becomes less powerful over time, since the pulsar in FRB 121102 is expected to be older than that of PTF10hgi.

We measure upper limits for the radio luminosity of the other nine SLSNe-I. In general, these limits favor models with faster spins, higher magnetic fields, larger ejecta mass, and significant free-free absorption. This is in contrast to the best model for PTF10hgi.

While there may well be multiple mechanisms to power SLSNe-I, the young pulsar model predicts an increase in flux for all SLSNe-I in this sample (Omand et al. 2018, C. M. B. Omand et al. 2019, in preparation). Repeating these observations with the same sensitivity in 5-10 yr would allow some constraint on pulsar parameters and six of them (SN2005ap, PTF09cnd, PTF09cwl, SN2010kd, SN2010gx, and SN2011ke) are predicted to be detectable under a range of scenarios. Observations today with more sensitive instruments (e.g., MeerKAT or SKA1) would also be likely to detect or better constrain the nature of the compact object.

PTF10hgi and the luminous radio source associated with FRB 121102 may be the first examples of $<100 \mathrm{yr}$ old pulsars (see, Gotthelf et al. 2000; De Luca 2017). Radio observations of SN1986J also imply the existence of a compact object of yet unknown nature (Bietenholz \& Bartel 2017a, 2017b, 2017c). Aside from their extreme luminosities, the former two sources are consistent with relatively flat radio spectra below $10 \mathrm{GHz}$. New observations of FRB 121102 would test whether the persistent radio source evolves in a similar manner as PTF10hgi. Similarly, broader spectral observations of PTF10hgi would test whether it has a similar spectral break as FRB 121102 (Chatterjee et al. 2017). 
If other pulsar-powered supernovae can be identified, the radio properties can be used to study the birth properties of pulsars. The radio measurements of PTF10hgi suggest it is powered by a pulsar born with a spin near the break-up period of $1 \mathrm{~ms}$. Meanwhile, Kashiyama \& Murase (2017) used the properties of FRB 121102 to estimate a birth spin period of $\lesssim \mathrm{a}$ few ms and age of 10-100 yr. However, estimates of birth spin period are somewhat degenerate with magnetic field, photon absorption processes, and more. Radio observations of known SLSN-I allow us to use the known age and optical light curve in modeling. Ultimately, we may be able to connect these young magnetars to the FRB phenomenon, which allows a host of new observational constraints, such as ejecta mass, age, and potentially spin period (Piro 2016).

We thank the VLA staff for their support of these challenging observations. This research made use of Astropy (https://www.astropy.org/), a community-developed core Python package for Astronomy (Astropy Collaboration et al. 2013; Price-Whelan et al. 2018).

C.J.L. acknowledges support under NSF grant 1611606 . K.M. acknowledges financial support from the Alfred P. Sloan Foundation and NSF grant PHY-1620777. K.K. acknowledges financial support from JSPS KAKENHI grant 18H04573 and 17K14248. C.M.B.O. has been supported by the Grant-in-aid for the Japan Society for the Promotion of Science (18J21778). S.B.-S, .K.A., and J.L. acknowledge support from NSF grant 1714897. T.J.W.L. acknowledges research carried out at the Jet Propulsion Laboratory, California Institute of Technology, under a contract with the National Aeronautics and Space Administration. The NANOGrav project receives support from National Science Foundation (NSF) Physics Frontier Center award number 1430284.

Facility: EVLA.

Software: rfpipe (Law 2017), astropy (Astropy Collaboration et al. 2013; Price-Whelan et al. 2018).

\section{ORCID iDs}

C. J. Law (i) https://orcid.org/0000-0002-4119-9963

K. Kashiyama (1) https://orcid.org/0000-0003-4299-8799

K. Murase (ib https://orcid.org/0000-0002-5358-5642

G. C. Bower (iD https://orcid.org/0000-0003-4056-9982

S. Burke-Spolaor (i) https://orcid.org/0000-0003-4052-7838

B. J. Butler (iD https://orcid.org/0000-0002-5344-820X

T. J. W. Lazio (i) https://orcid.org/0000-0002-3873-5497

\section{References}

Astropy Collaboration, Robitaille, T. P., Tollerud, E. J., et al. 2013, A\&A, 558, A33

Bietenholz, M. F., \& Bartel, N. 2017a, ApJ, 839, 10

Bietenholz, M. F., \& Bartel, N. 2017b, ApJ, 851, 7

Bietenholz, M. F., \& Bartel, N. 2017c, ApJ, 851, 124

Bower, G. C., Metzger, B. D., Cenko, S. B., Silverman, J. M., \& Bloom, J. S. 2013, ApJ, 763, 84

Brown, P. J., Breeveld, A. A., Holland, S., Kuin, P., \& Pritchard, T. 2014, Ap\&SS, 354, 89

Chatterjee, S., Law, C. J., Wharton, R. S., et al. 2017, Natur, 541, 58

Coppejans, D. L., Margutti, R., Guidorzi, C., et al. 2018, ApJ, 856, 56
Cordes, J. M., \& Chatterjee, S. 2019, ARA\&A, 57, 417

Cordes, J. M., \& Lazio, T. J. W. 2002, arXiv:astro-ph/0207156

De Luca, A. 2017, JPhCS, 932, 012006

Eftekhari, T., Berger, E., Margalit, B., et al. 2019, ApJL, 876, L10

Elvis, M., Wilkes, B. J., McDowell, J. C., et al. 1994, ApJS, 95, 1

Gaensler, B. M., \& Slane, P. O. 2006, ARA\&A, 44, 17

Gajjar, V., Siemion, A. P. V., Price, D. C., et al. 2018, ApJ, 863, 2

Gal-Yam, A. 2012, Sci, 337, 927

Gal-Yam, A., Mazzali, P., Ofek, E. O., et al. 2009, Natur, 462, 624

Gotthelf, E. V., Vasisht, G., Boylan-Kolchin, M., \& Torii, K. 2000, ApJL, 542, L37

Gruzinov, A. 2005, PhRvL, 94, 021101

Guillochon, J., Parrent, J., Kelley, L. Z., \& Margutti, R. 2017, ApJ, 835, 64

Hatsukade, B., Tominaga, N., Hayashi, M., et al. 2018, ApJ, 857, 72

Inserra, C., Smartt, S. J., Jerkstrand, A., et al. 2013, ApJ, 770, 128

Kasen, D., \& Bildsten, L. 2010, ApJ, 717, 245

Kashiyama, K., \& Murase, K. 2017, ApJL, 839, L3

Kashiyama, K., Murase, K., Bartos, I., Kiuchi, K., \& Margutti, R. 2016, ApJ, 818,94

Keane, E. F., \& Petroff, E. 2015, MNRAS, 447, 2852

Kulkarni, S. R., Ofek, E. O., Neill, J. D., Zheng, Z., \& Juric, M. 2014, ApJ, 797, 70

Law, C. J. 2017, rfpipe: Radio interferometric transient search pipeline, Astrophysics Source Code Library, ascl:1710.002

Law, C. J., Abruzzo, M. W., Bassa, C. G., et al. 2017, ApJ, 850, 76

Law, C. J., Bower, G. C., Burke-Spolaor, S., et al. 2018, ApJS, 236, 8

Leloudas, G., Chatzopoulos, E., Dilday, B., et al. 2012, A\&A, 541, A129

Liu, L.-D., Wang, S.-Q., Wang, L.-J., et al. 2017, ApJ, 842, 26

Lunnan, R., Chornock, R., Berger, E., et al. 2014, ApJ, 787, 138

Maeda, K., Tanaka, M., Nomoto, K., et al. 2007, ApJ, 666, 1069

Margalit, B., \& Metzger, B. D. 2018, ApJL, 868, L4

Margalit, B., Metzger, B. D., Berger, E., et al. 2018, MNRAS, 481, 2407

McMullin, J. P., Waters, B., Schiebel, D., Young, W., \& Golap, K. 2007, in ASP Conf. Ser. 376, Astronomical Data Analysis Software and Systems XVI, ed. R. A. Shaw, F. Hill, \& D. J. Bell (San Francisco, CA: ASP), 127 Merloni, A., Heinz, S., \& di Matteo, T. 2003, MNRAS, 345, 1057

Metzger, B. D., Berger, E., \& Margalit, B. 2017, ApJ, 841, 14

Metzger, B. D., \& Bower, G. C. 2014, MNRAS, 437, 1821

Metzger, B. D., Margalit, B., Kasen, D., \& Quataert, E. 2015, MNRAS, 454, 3311

Metzger, B. D., Margalit, B., \& Sironi, L. 2019, MNRAS, 485, 4091

Milisavljevic, D., Patnaude, D. J., Chevalier, R. A., et al. 2018, ApJL, 864, L36 Murase, K., Kashiyama, K., Kiuchi, K., \& Bartos, I. 2015, ApJ, 805, 82 Murase, K., Kashiyama, K., \& Mészáros, P. 2016, MNRAS, 461, 1498 Nicholl, M., Guillochon, J., \& Berger, E. 2017, ApJ, 850, 55

Nicholl, M., Smartt, S. J., Jerkstrand, A., et al. 2015, MNRAS, 452, 3869 Omand, C. M. B., Kashiyama, K., \& Murase, K. 2018, MNRAS, 474, 573

Pastorello, A., Smartt, S. J., Botticella, M. T., et al. 2010, ApJL, 724, L16

Petroff, E., Barr, E. D., Jameson, A., et al. 2016, PASA, 33, e045

Petroff, E., Hessels, J. W. T., \& Lorimer, D. R. 2019, A\&ARv, 27, 4

Piro, A. L. 2016, ApJL, 824, L32

Prajs, S., Sullivan, M., Smith, M., et al. 2017, MNRAS, 464, 3568

Price-Whelan, A. M., Sipőcz, B. M., Günther, H. M., et al. 2018, AJ, 156, 123 Prochaska, J. X., \& Zheng, Y. 2019, MNRAS, 485, 648

Quimby, R. M., Aldering, G., Wheeler, J. C., et al. 2007, ApJL, 668, L99

Quimby, R. M., Kulkarni, S. R., Kasliwal, M. M., et al. 2011, Natur, 474, 487

Reines, A. E., Greene, J. E., \& Geha, M. 2013, ApJ, 775, 116

Schulze, S., Krühler, T., Leloudas, G., et al. 2018, MNRAS, 473, 1258

Soderberg, A. M., Frail, D. A., \& Wieringa, M. H. 2004, ApJL, 607, L13

Spitkovsky, A. 2006, ApJL, 648, L51

Tanaka, S. J., \& Takahara, F. 2010, ApJ, 715, 1248

Tanaka, S. J., \& Takahara, F. 2013, MNRAS, 429, 2945

Tauris, T. M., \& Manchester, R. N. 1998, MNRAS, 298, 625

Tchekhovskoy, A., Spitkovsky, A., \& Li, J. G. 2013, MNRAS, 435, L1

Tendulkar, S. P., Bassa, C. G., Cordes, J. M., et al. 2017, ApJL, 834, L7

Vinko, J., Zheng, W., Pandey, S. B., et al. 2012, AAS Meeting , 219, 436.04

Vreeswijk, P. M., Savaglio, S., Gal-Yam, A., et al. 2014, ApJ, 797, 24

Watts, A. L., Andersson, N., Chakrabarty, D., et al. 2016, RvMP, 88, 021001

Woosley, S. E., \& Bloom, J. S. 2006, ARA\&A, 44, 507

Yaron, O., \& Gal-Yam, A. 2012, PASP, 124, 668 